\title{
External Mass Transfer of a Single Particle in Nonlinear Extensional Flow
}

\author{
Anjun Liu ${ }^{1,2}$, Jie Chen ${ }^{2,3 *}$, Zhenzhen Wang ${ }^{2,3}$, Chao Yang ${ }^{2,3 *}$ and Zai-Sha Mao ${ }^{2}$ \\ ${ }^{1}$ School of Chemical Engineering and Technology, Tianjin University \\ Tianjin 300350, China \\ ${ }^{2}$ CAS Key Laboratory of Green Process and Engineering, Institute of Process Engineering, Chinese Academy of Sciences \\ Beijing 100190, China \\ jchen@ipe.ac.cn; chaoyang@ipe.ac.cn \\ ${ }^{3}$ University of Chinese Academy of Sciences \\ Beijing 100049, China
}

\section{Extended Abstract}

The fundamental research on the transfer phenomena (momentum, heat and mass transfer) of a single particle is of great importance in practical application in chemical and processing industry. The nonlinear flow that is produced in industrial rotary devices is very common and plays important roles on the particle transfer phenomena. Unfortunately, most works neglecting the effect of nonlinear term are dedicated to study a single particle (bubble, liquid drop, or solid particle) immersed in a simplified linear flow, such as in simple shear flow or in simple extensional flow. In order to explore the nonlinear problem, Favelukis [1,2] theoretically analysed the flow field and external mass transfer of a single spherical particle in the nonlinear extensional flow field. However, Favelukis [2] just explored the problem at large Peclet $(P e>>1)$ numbers. So, this numerical work firstly verified Favelukis' [2] theoretical formula and then studied the external mass transfer of a spherical particle in the uniaxial and biaxial nonlinear extensional flows in a more wide range of $1 \leq P e \leq 100000,-5 \leq E$ (the nonlinear intensity of the extensional flow) $\leq 10$.

For all Peclet numbers, the simulation results show that the mass transfer rate of the nonlinear case is lower than that of the linear case in the range of $-1.04<E<0$ (bubble and droplet) and $-0.49<E<0$ (solid particle). Outside these ranges, the mass transfer rate is enhanced by the nonlinear flow.

For a spherical droplet/bubble in the non-linear flow, the simulated Sherwood number $(S h)$ absolutely agrees with the Favelukis' [2] theoretical results at the convection-dominated cases (Pe>1000). For the diffusion-dominated cases and the transitional zone $(1 \leq P e \leq 1000)$, the empirical correlations of the external mass transfer rate of a single spherical bubble or droplet in the nonlinear extensional flow which Favelukis [2] cannot solve theoretically are proposed to predict $S h$ in the range of $-5 \leq E \leq 10$.

But for that of a spherical solid in the non-linear flow, when $E>5 / 4$, the simulated $S h$ of a solid sphere in the nonlinear extensional flow is larger than the results given by Favelukis [2]. The reason is the effect of vortex formed outside particles on mass transfer, which is considered in this work. And $S h$ was provided in an extended range $(-5 / 17 \leq E \leq 5 / 4, E \neq 0)$ that Favelukis [2] did not list as a theoretical correlation. In addition, the empirical correlations of the external mass transfer of a single solid sphere are proposed to predict $S h$ in the whole range of $P e(1 \leq P e \leq 100000)$.

Acknowledgment: This work was supported by the National Key Research and Development Program (2016YFB0301701), National Natural Science Foundation of China (21606234, 21490584) and Key Research Program of Frontier Sciences of CAS (QYZDJ-SSW-JSC030).

\section{References}

[1] M. Favelukis, “A drop in uniaxial and biaxial nonlinear extensional flows,” Phys Fluids, vol. 29, no. 087102, 2017.

[2] M. Favelukis, "Mass transfer around bubbles, drops, and particles in uniaxial and biaxial nonlinear extensional flows," AIChE J., vol. 65, no. 1, pp. 398-408, 2018. 